Proc. 21st Winter Workshop on

Nuclear Dynamics (2005) 000-000

22st Winter Workshop

on Nuclear Dynamics

San Diego, California, USA

March 12-18, 2006

\title{
Resonance Production in RHIC Collisions
}

\author{
C. Markert for the STAR collaboration ${ }^{1}$ \\ ${ }^{1}$ Physics Department, Kent State University, Kent, OH 44242, USA
}

\begin{abstract}
Results of resonance particle production measured at RHIC in $\sqrt{s_{\mathrm{NN}}}=200 \mathrm{GeV} \mathrm{Au}+\mathrm{Au}$ collisions are compared to measurements in $\mathrm{p}+\mathrm{p}$ and $\mathrm{d}+\mathrm{Au}$ collisions in order to verify the existence of an extended hardronically interacting medium. Yield and momentum distributions of resonances maybe modified during the fireball lifetime due to resonance decay and the subsequent rescattering of their decay daughters as well as the regeneration of resonances from their decay products. Modified momentum spectra in heavy ion collisions may change the nuclear modification factor $\mathrm{R}_{\mathrm{AA}}$. The influence on the elliptic flow $\mathrm{v}_{2}$ due to late regeneration of resonances is discussed.
\end{abstract}

Keywords: resonance, lifetime, strange, freeze-out, medium PACS: $25.75 . \mathrm{Dw}$

\section{Introduction}

If the life time of the hadronic medium created in a heavy ion reaction is long enough (on the order of a few $\mathrm{fm} / \mathrm{c}$ ) resonances decay inside the medium and their decay product can therefore interact with the medium as well. In a dense hadronic medium resonances can also be regenerated from their decay products. This is a dynamical process of creating and decaying resonances (detailed balance). The measured resonances are therefore a composition of early and late produced and decayed resonances. The largest fraction comes from the late decay since the probability of another interaction of the decay particles from early decays is larger than from late decays. Therefore by using the yield of resonances and knowing their decay, re-scattering and regeneration cross section we are able to estimate the lifetime of the hadronic phase.

A resonance signal loss in the low momentum region is caused by a larger re-scattering than regeneration cross section. This will also influence the low momentum region of the nuclear modification factor $\mathrm{R}_{A A}$ spectrum, which is the ratio of the heavy ion transverse momentum spectrum divided by the $\mathrm{p}+\mathrm{p}$ spectrum scaled by the number of binary collisions. 
More than $60 \%$ of the stable particles originate from weak and resonance decays. This raises the question how the elliptic flow $\mathrm{v}_{2}$ of stable particles is affected if regeneration of resonances after chemical freeze-out is present. Furthermore recombination models predict an enhancement of $\mathrm{v}_{2}$ for resonances due to the larger $\mathrm{v}_{2}$ contribution from regenerated resonances. The yield would scale according to constituent quark scaling of the recombining hadrons, e.g. $\mathrm{K}+\pi \rightarrow \mathrm{K}(892)(2+2=4)$ and $\Lambda+\pi \rightarrow \Sigma(1385)(3+2=5)$. The observed enhancement of $\mathrm{v}_{2}$ would depend on the fraction of regenerated resonances [1].

\section{Rescattering and Regeneration Cross Sections}

Figure 1 shows the resonance to stable particle ratios for $\mathrm{p}+\mathrm{p}$ and $\mathrm{Au}+\mathrm{Au}$ collisions systems [2, 3]. The ratios are normalized to unity for $p+p$ collisions. The deviation of the ratio from unity in $\mathrm{Au}+\mathrm{Au}$ collisions indicate a late hadronically interacting medium where decay of resonances and the rescattering of the decay particles is larger than the regeneration of resonances. A ranking of the overall regeneration over rescattering cross section can be deduced as follows: $R_{\Lambda(1520)}<R_{K(892)}<$ $R_{\Sigma(1358)}$

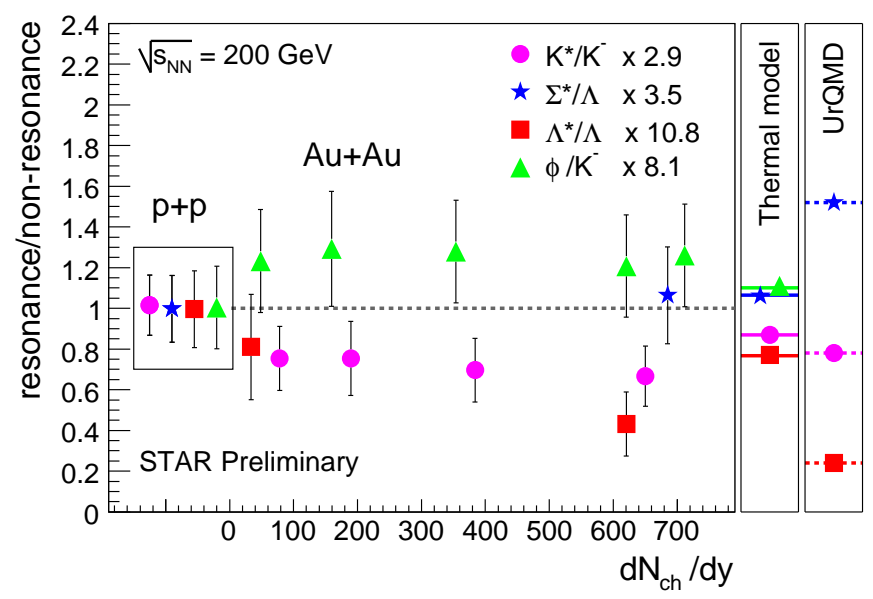

Fig. 1. Resonance to stable particle ratios of $\Sigma(1385) / \Lambda, \phi / K^{-}, \mathrm{K}(892) / \mathrm{K}^{-}$and $\Lambda(1520) / \Lambda$ for $p+p$ and $A u+A u$ collisions at $\sqrt{s_{N N}}=200 \mathrm{GeV}$. The ratios are normalized to unity in $p+p$ collisions. The quadratic sum of statistical and systematic uncertainties are included in the error bars. Thermal and UrQMD model predictions are presented as well [4, 5, 6].

Sascha Vogel showed at this workshop a microscopic model calculation (UrQMD) of the regeneration cross sections of resonances and confirms the ranking as derived from the data [7]. This model predicts a signal loss in the low momentum region due 
to rescattering. A comparison of the transverse momentum spectrum of $\mathrm{p}+\mathrm{p}$ and $\mathrm{Au}+\mathrm{Au}$ collisions using the nuclear modification factor $\mathrm{R}_{\mathrm{AA}}$ shows for the $\mathrm{K}(892)$ a larger suppression in the momentum region from $\mathrm{p}_{\mathrm{T}}=0-2 \mathrm{GeV}$ compared to the long lived $\phi$ and the $\mathrm{K}_{\mathrm{S}}^{0}$ (Figure 21). These data support the concept of a hadronic interacting medium after chemical and before kinetic freeze-out which changes the measured resonance yield and the momentum spectra. Thermal model predictions by $\mathrm{W}$. Florkowski et. al show a shift to higher values compared to the measured $\mathrm{K}(892)$ transverse momentum distribution in the low momentum region [8]. Therefore nuclear suppression factors $\left(\mathrm{R}_{A A}\right)$ of resonances are not directly comparable to stable particles as long as the momentum dependent signal loss of resonances in the hadronic phase is not taken into account.

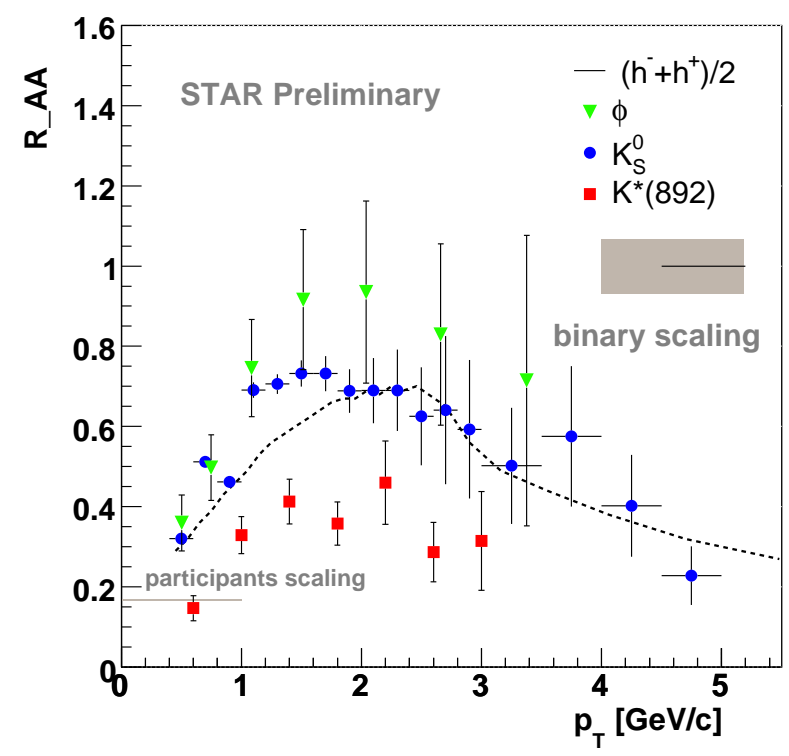

Fig. 2. The ratio of the transverse momentum of $\mathrm{Au}+\mathrm{Au}$ divided by $\mathrm{p}+\mathrm{p}$ collisions normalized to the number of binary collisions.

The low mean transverse momentum for heavy multi strange particles ( $\Xi$ and $\Omega$ ) in $\mathrm{Au}+\mathrm{Au}$ collisions was interpreted as a sign of early decoupling from the hadronic system at high temperature and low transverse velocity. In Figure 3 the $\Sigma(1385)$ shows the same trend as $\Xi$ and $\Omega$. Since the regeneration cross section of the $\Sigma(1385)$ from $\pi+\Lambda$ is very large [ 7] we expect to measure more late produced $\Sigma(1385)$, i.e. resonances produced close to the kinetic freeze-out. This would suggest a late decoupling of the $\Sigma(1385)$ from the hadronic phase, and thus possibly contradict the simple connection between $\left\langle p_{T}\right\rangle$ and the decoupling parameters. Based on the 
primordial $\Lambda$ spectra, a $\Lambda$ from a $\Sigma(1385)$ decay is not expected to exhibit much early decoupling. Thus it is important to measure the $\Xi(1530)$ resonance in heavy ion collisions, verify its regeneration cross section and estimate the contribution of $\Xi$ 's from the $\Xi(1530)$ decays.

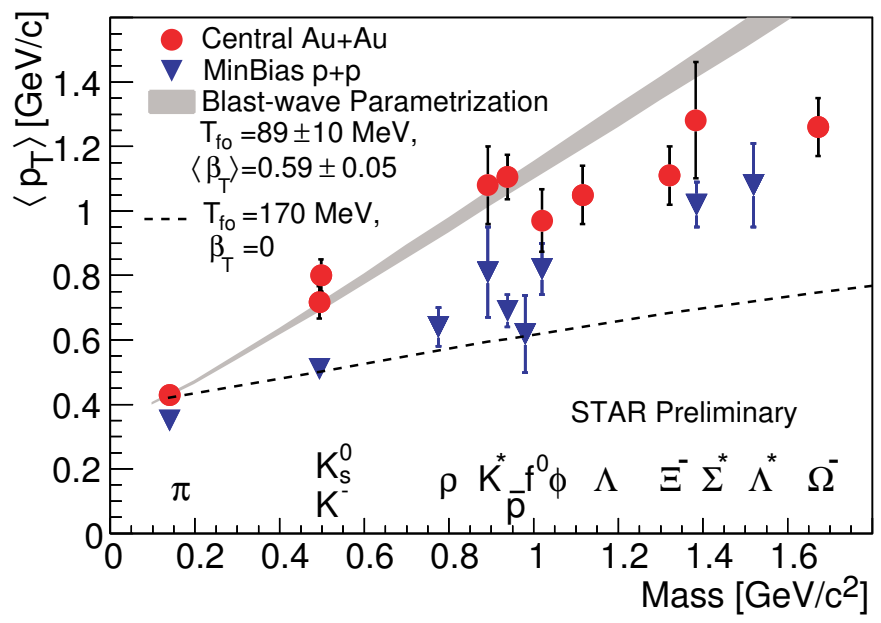

Fig. 3. The $\left\langle p_{T}\right\rangle$ vs particle mass measured in min-bias $p+p$ and most central $A u+A u$ collisions at $\sqrt{s_{N N}}=200 \mathrm{GeV}$. Statistical and systematic uncertainties are shown [2, 3].

\section{3. $\mathrm{d}+\mathrm{Au}$ Collisions}

Since no extended hadronic medium for $\mathrm{d}+\mathrm{Au}$ collisions is created, the momentum distribution of $\mathrm{p}+\mathrm{p}$ and $\mathrm{d}+\mathrm{Au}$ collisions for resonances are expected to be similar compared to the stable particles. Figures 4 shows the preliminary ratio of the transverse momentum spectra measured in $\mathrm{d}+\mathrm{Au}$ divided by $\mathrm{p}+\mathrm{p}$ spectra normalized to the number of binary collisions. The $\mathrm{R}_{d A u}$ of $\Sigma(1385)$ seams to follow the proton $\mathrm{R}_{d A u}$ (right), while the $\mathrm{K}(892)$ deviates from the $\mathrm{K}$ and $\pi$ in the low momentum region. This result is unexpected, if we assume no rescattering in $\mathrm{d}+\mathrm{Au}$. The data have to be further investigated and the yields have to be checked for consistency.

\section{Elliptic Flow $\mathrm{v}_{2}$}

Anisotropic flow results are often used as a strong evidence for the formation of the QGP in $\mathrm{Au}+\mathrm{Au}$ Collisions at RHIC. The magnitude and centrality dependence of the elliptic flow $\mathrm{v}_{2}$ is used as a proof of early thermalization. The so-called "mass splitting", the characteristic dependence of $\mathrm{v}_{2}\left(\mathrm{p}_{\mathrm{T}}\right)$ on the particle mass, is well described when using a QGP Equation of State. In addition the constituent 

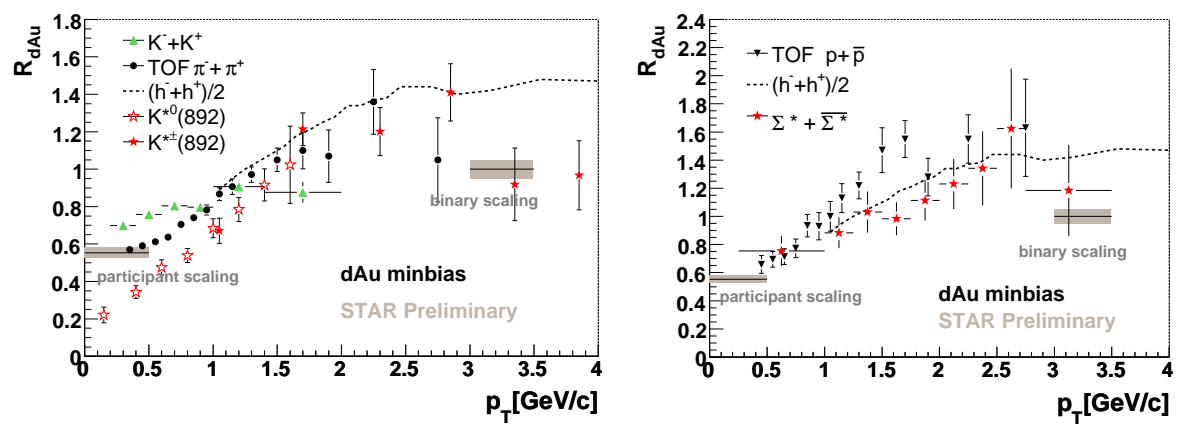

Fig. 4. The ratio of the transverse momentum of $d+A u$ divided by $p+p$ collisions normalized to the number of binary collisions for meson (left) and baryons (right).

quark scaling in the intermediate transverse momentum region is often cited as a proof of deconfinement and partonic (pre-hadronic) collectivity. One therefore would like to study the elliptic flow of resonances in order to test the probability of formation at the early stage of the collision and the effect the expansion dynamics of the source including rescattering and regeneration of resonances will have on their $\mathrm{v}_{2}$. The elliptic flow of the long lived $\phi$ meson resonance shows the expected mass dependence in the low momentum region and a constituent quark scaling similar to the $\mathrm{K}_{S}^{0}$ meson in the intermediate transverse momentum region. In the near future we will analyze the elliptic flow of the short lived $\mathrm{K}(892)$ and $\Delta(1232)$ resonances.

\section{Conclusions}

Resonances are a unique tool to probe the hadronic medium in heavy ion collisions. It allows us to estimate the lifetime of the hadronically interacting medium and derive the rescattering over regeneration cross section ranking for different resonances. Using a microscopic models actual regeneration cross sections can be determined based on the data. Further more we gain in a better understanding of the hadronic interaction probabilities which helps us to distinguish between early and late decoupling of particle species from the hadronic medium.

\section{Acknowledgments}

I would like to thank Sascha Vogel and Marcus Bleicher for their detailed UrQMD analysis to get a better understanding of hadronic cross sections in terms of resonance regeneration. And I also would like to thank the STAR collaboration for the support in presenting this data. 


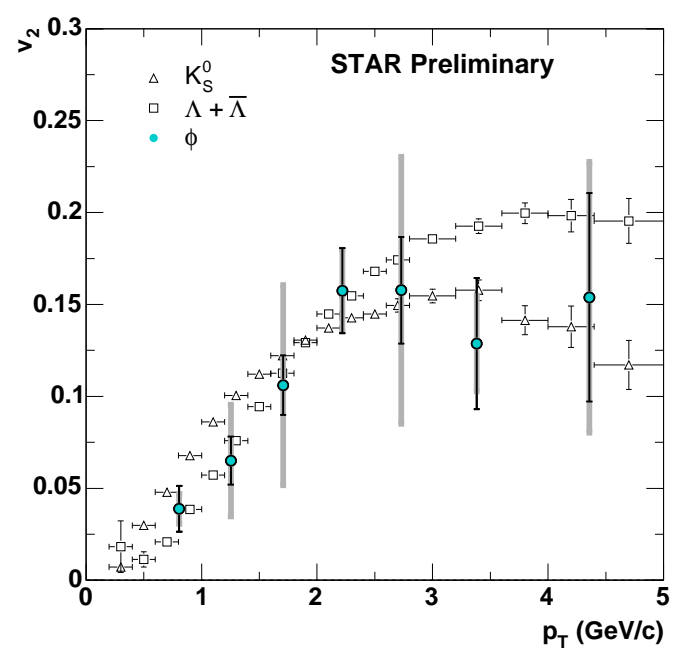

Fig. 5. The $\mathrm{v}_{2}$ of $\phi$ meson compared to $\mathrm{K}_{S}^{0}$ meson and $\Lambda$ baryon as a function of $\mathrm{p}_{\mathrm{T}}$. Statistical and systematical errors are included [10].

\section{References}

1. C. Nonaka et. al., Phys.Rev. C69 (2004) 031902, nucl-th/0312081.

2. J. Adams, et al., (STAR collboration), Subm. to Phys. Rev. Lett, nucl-ex/0604019

3. Sevil Salur, PhD Thesis Yale University 2006.

4. F. Becattini, Nucl. Phys. A702, 336 (2002).

5. M. Bleicher et al., Phys. Lett. B530, 81 (2002).

6. M. Bleicher and H. Stöcker, J. Phys. G30 (2004) 111.

7. S. Vogel, These proceedings.

8. W. Florkowski, W. Broniowski and P. Bozek, J.Phys. G30 (2004) 1321.

9. S.Salur (for the STAR Collaboration) nucl-ex/0606002.

10. X. Cai for the STAR collaboration, Quark Matter 2005, Budapest, Hungary, 4-9 Aug 2005. e-Print Archive: nucl-ex/0511004 